\title{
An Extensive Study on Gravitational Search Algorithm
}

\author{
*C. Venkateswaran, M. Ramachandran, Sathiyaraj Chinnasamy, Chinnasami Sivaji, M. Amudha
}

Management Studies department, Maharishi Markandeshwar (Deemed to be University), Ambala, Haryana. India

REST Labs, Kaveripattinam, Krishnagiri, Tamil Nadu, India.

*Corresponding author Email: venky.professor@gmail.com

\begin{abstract}
Gravitational search algorithm is a naturally occurring algorithm based on Newton's mathematical model of the law of gravitation and motion. Over the course of a decade, researchers have provided many variants of the gravitational search algorithm by modifying its parameters to effectively solve complex optimization problems. This paper conducts a comparative analysis of ten types of gravity search algorithms that modify the three parameters of optimum, speed and position. Tests are conducted on two sets of benchmark types, namely standard functions and issues belonging to different types such as CEC2015 functions, univocal, multimodal and unrestricted optimization functions. Performance comparison is evaluated and statistically validated based on the average exercise value and concentration graph. In trials, IGSA has achieved excellent accuracy through a balanced trade between exploration and exploitation. Furthermore, three negative breast cancer datasets were considered to analyze the efficacy of GSA variants for the black section. Different performance analyzes were performed based on both quality and quantity with the integrated jacquard index as a performance measure. Tests confirm that the IGSA based method worked better than other methods.

Keywords: Optimization, Gravitational search algorithm, Multi-objective Gravitational Search Algorithm (MOGSA), Adaptive gravitational search algorithm, Binary gravitational search algorithm, Disruption based gravitational search algorithm
\end{abstract}

\section{Introduction}

Optimization is the process of continuous training of the model resulting in maximum and minimum performance evaluation. This is one of the most important milestones in machine learning to get the best results. In this article, we will discuss important optimization algorithms. The Gravity Search Algorithm (GSA) is one of the latest natural-inspired algorithms with advanced problem-solving capabilities. GSA was inspired by Newton's law of gravitation and the laws of motion. The purpose of the paper is to explore the application of GSA to various optimization issues. Sheet GSA and previous GSA based optimization Provides a brief overview of jobs. In terms of literature, GSA is highly accurate for most optimization issues, Able to provide effective and robust high quality solution. The main problem with proposing a multipurpose variant for the GSA, the renewal of the particle mass based on the value of multiple objectives. In MOGSA, The mass of all the moving particles is set together; the mass of the archived particles is updated at objective intervals based on distance. The neighboring neighbors. However, no equation is given to relate Mass value to distance value. This technique is similar to the technique of knocking down archived components Distributes uniformly. They are by forces used by archived members moving to new levels.

\section{Optimization}

The Various optimization and in engineering application issues GSA is widely used. Whether GSA is an effective and reliable method these results have been proven. However, its performance is defined by its potential mechanism, which is likely to result in a pre-integration and search capability is low. This is because the GSA uses interactive information between individuals in the entire population to continuously create new individuals, whereas it is difficult for individuals to escape from the local peak if they are stuck in a predicament [1]. Besides, the movement of individuals Speeds pulls and depends on the masses during the entire search process. Late in the search process, individuals' refresh speeds are so small that it results in less exploitative behavior to find a better solution, indicating that an inefficient upgrade function is being used to further enhance the search space[2]. Therefore, it is useful and valuable to balance GSA's search capability between exploration and exploitation, in order to improve its performance from the beginning to the end of the optimization process. In view of these two shortcomings, a hierarchical demographic system is adopted to mitigate them and improve the GSA's search performance [3]. The three rules (immigration, local optimization and global optimization) are established based on a number of empirical optimization strategies that improve GA's local optimization efficiency and its integration speed. Utilizes the generation alternative model based on the improved algorithm, minimum generation spacing and hybrid crossover operators (BLX- $\alpha$ )[4]. This proposed method not only has the advantage of having simple algorithms and good flexibility, planar straight forwardness and spatial straightforward estimates. Adjusting shortcut and mutation rates using the mean and constant deviations of fitness values for each generation and optimal results can be achieved with adaptive GAs with decreasing mutation rate and increasing crossover rate [5]. Based on the results, it has been decided that SGSA is capable of exploration and exploitation, while the proposed algorithm (IGSA) enhances SGSA's exploration and exploitation for high-dimensional immoral and multimodal optimization functions. We used IGSA's high potential for high- 
dimensionality operations to find the best solutions [6]. Two possible values for some optimization issues, there are variables with zero and one. These issues are handled by binary versions. Introduced the Binary Gravity Search Algorithm (PGSA). In the binary context, the equations for key and speed upgrades same as in the sequel, the humming distance is also used to calculate the JRP distance [7]. A control optimization problem mathematically defined as Eq. Under the Ng number of equation control functions Development of an objective process and expressed by $\mathrm{g}$ and $\mathrm{h}$ in the equation this problem is caused by the $\mathrm{Nh}$ number of equation control functions. To deal with restrictions, it is common practice to impose punishment for objective action [8]. To evaluate Solution $\mathrm{X}$ in the mitigation problem. The problem in identifying a system parameter, which can be described as the function of a set of parameters, is the problem in obtaining the best set of possible parameter values by minimizing the objective function. Objective function is defined as the function of errors between the rated outputs of the model and the actual ones. Therefore, the parameter identification problem can be considered as an improvement problem, and the use of a better optimization algorithm is an important method [9]. Non-linear systems have been shown to be effective in dealing with parameter identification. However, as reported in some of GA's shortcomings, There are many more to overcome and Due to the loss of population diversity at a sub-point Premature integration is one of the main problems. It can track the local minima so that the best solution cannot be obtained, In a word; the search capability of the performance determines the ability to solve Identification of a complex system [10].

\section{Gravitational search algorithm}

Gravitational search algorithm (GSA) is a new heuristic search algorithm, which was first introduced by Rashedi et al. where it is constructed based on the law of Gravity and the notion of mass interactions. Gravity force is a universe attracts other particle with a force that is directly proportional. In an earlier version of the GSA, the algorithm "gravity keys" Multidimensional continuous space (search location) Causing direct changes in the position of search points [11]. The effect of these keys is on the binary vector Converts the probability value to each element, it guides those elements. Get a value of 0 or 1 [1]. Gravitational search algorithm (GSA) In terms of the metaphor of gravity and mass interactions Based on the newly created population is a random optimization system [12]. This approach provides a functional mechanism in physics that reflects mass interactions under the influence of gravity and moves through a multi-dimensional search space. In this method, the particles are treated as objects and their efficiency is measured by their mass. Each particle represents a candidate solution to a search problem [13]. All particles are attracted to each other by the force of gravity, and this force causes the universal motion of all objects towards heavier objects. Therefore, masses cooperate with each other using direct communications by the force of gravity [14]. The gravitational search algorithm is a recent natural-inspired algorithm proposed to solve upgrade Problems based on the law of gravity. Many researchers point to a greater number of problems Used the gravity search algorithm, because this requires only two parameters and the ability to find the optimal solution globally and Provides better results compared to other nature inspired methods [15]. Explains the applications of the gravity search algorithm in different domains. The purpose of this study is to improve the complexity (gravity search algorithm), Is to provide a comprehensive survey of researchers in the field of mass intelligence and naturally inspired algorithms. The first purpose of this paper is to solve optimization problems Determine the number of methods derived from the original GSA, As well as design recognition, power system and Using the Gravity Search Algorithm in various fields such as data [16]. How many methods are proposed using mining, taxonomy \& clustering and gravity search algorithm and other naturally study is to explore the importance of the gravity. New Holistic Newton's gravitation and motion. In the GSA, agents are treated as substances and their efficiency is measured by their mass [17]. All these objects are attracted to each other by the force of gravity, and this force causes the universal motion of all objects towards heavier objects. So masses cooperate using direct contact by the force of gravity. Heavy masses similar to good solution move slower than lighter ones, this guarantees the exploitation step of the algorithm [18]. In the GSA, each mass (agent) It has four specifications: static, passive mass, and active Gravitational mass and passive gravitational mass. The position of the mass is similar to the solution of the problem And its gravitational and passive masses Are determined using an exercise function [19]. In other words, every mass Provides a solution and is guided by the algorithm correct adjustment of the gravitational and inertial masses. Over time, the masses are expected to Inspired by the heavier mass. This mass search space providing an optimal solution. The GSA can be considered an isolated entity of the masses. This is Newton's gravity and the masses in accordance with the rules of motion Like a little artificial world. People obey the following laws [20].

\section{Multi-objective Gravitational Search Algorithm (MOGSA)}

MOGSA appears to be comparable or better performing when considering the other three. As can be easily guessed from, for MOP6, the GD factor in MOGSA outperforms the scale of the other three best GD metrics, and the S factor is greatly improved in addition to MOGA2. Slightly better spacing factor. Because of the use of MOGSA gravitational fields, the particles are attracted to the heavier masses around the barrette frontal area that are not uniformly distributed (thus the masses are thus defined) thus creating a better distribution of solutions [21]. Without using any inching method directly. Finally, it should be noted that although gravity-based methods have proven their effectiveness, the time taken to implement a iteration in MOGSA is slightly longer than other conventional methods such as PSO. K- Decreases at best time. As a future job, one can introduce the concept of ambiguous multi-purpose gravity search algorithm (FMOGSA) [22]. Ambiguity can be used before Barreto, meaning that each solution in the archive will have a number of members, indicating the extent to which solutions are considered non-dominant. MOGSA for each given, GA and ACO. In the order of the final stacking For the 
purpose of describing the material of each layer, Glass layers are denoted by blank numbers, at the same time the carbon layers are denoted by underlined numbers [23]. Number of layers and materials there are two main factors in multi-purpose optimization. In addition, the angles of the composite layers play a role in determining the first natural frequency. If the value of the weight factor $(\alpha)$ is set to zero, the complexity of reducing cost is reduced to a single-purpose optimization complex. Thus, MOGSA can be used with laminated layers entirely of fiberglass [24]. All application modes, including GA, ACO and MOGSA, reach the best designs, with carbon-epoxy-based coatings on the exterior and glass-epoxy-on-the-interior. This creates a sandwich-type mix, Placed outside their Structural function is ensured by hard carbon layers, for the flexibility of the laminate there contribution will be maximum, At the same time the inner layers are used only to increase the distance. Reduces outer layers and total cost from neutral aircraft [25]. To evaluate the performance of our algorithm, in each test operation With 25 independent runs we used all the comparison methods. SPEA2, TV-PSO and CA-MOGSA NSGA-II Of results between four comparable algorithms Minimum (min), maximum (maximum), average and show constant deviation (std). MOGSA and NSGSA are Generative Distance (GD), respectively [26]. Accumulation (C) measurements, spacing (SP) and Based on maximum amplitude (MS). Also, FON as selected functions And shows the box plots of ZDT2. From, the performance of the proposed CA-MOGSA in terms of GD and C concentric measurements is much better than that of MOGSA, TVPSO and NSGSA and comparable to modern EAs [27]. Namely, NSGA-II and SPEA2. illustrate that for CUR, FON, and POL functions, CA-MOGSA can provide an approximate pareto-optimal set with lower GD and C values than other comparative algorithms. -MOGSA for ZDT1 and ZDT2 functions. The archive size is set to 50 for MOGSA, SPEA2, TV-PSO and up to 50 for CA-MOGSA. The cross-section and mutation probabilities are set to 0.9 and 0.1 for SPEA2 and NSGA-II, respectively, and the mutation ratios are set to 0.2 and 0.1 for TV-PSO and MOGSA, respectively [28]. Other parameters are set as given in the literature [94]. GSA is derived from the general law of gravitational force is a new multiagent optimization algorithm. Algorithm some particles are influenced by gravitational forces Based on the bottom movement, Used by others [29]. Hasanzade and Romani are the first in the GSA Proposed a multi-purpose variant; this is called Multi-Objective GSA (MOGSA). Save solutions that are not dominated by MOGSA Uses external archive and Simple as Canyon proposed to update the external archive Such as Multi-Objective PSO (SMOPSO) uses the same concept. For this purpose, of each objective function the space is divided into equal sections, the result is a full performance gap Divided into hyper-rectangles [30]. When the number of archived members exceeds the maximum archive length, of the most congested hyper-rectangle A member will be randomly selected and removed. The main problem with proposing a multi-purpose variant for the GSA, the renewal of the mass of particles based on the value of multiple purposes. In MOGSA, the mass of all moving particles is set together; The mass of the archived particles was updated at objective intervals based on distance. Neighboring neighbors. However, no equation has been given to relate mass value to distance value [31].

\section{Adaptive gravitational search algorithm}

Approximate algorithms designed to be used for med heuristic, engineering problems, including the Adaptive Gravity Search Algorithm method. It is clearly desirable that these algorithms be applied to real optimization problems without the need for very skilled labor. To date, however, adjusting the parameters for their application requires considerable time and effort, and therefore, from an engineering perspective, it is desirable to add strength and compatibility to these methods [32]. The latter adaptive characteristic is particularly important from the point of view of practical applications. To add adaptability to an optimization algorithm one must understand two significant relationships. One, the parameters and The quality between the behavior of the algorithm And quantity is the analysis of the relationship [33]. The other, the success of the search for the behavior of the algorithm or an analysis of the quality and quantity relationship between failure. The change in the algorithm due to the results of this analysis Should be carefully weighed, thus associated with the success of the search An optimal algorithm can determine behavior again helps in maintaining the search conditions. This behavior is understandable [34]. The search process in GSAs depends on the number of $\mathrm{N}$ agents, the maximum number of repetitions kmax, the gravitational constant $\mathrm{g}(\mathrm{k})$ and the parameter that has a positive effect on the results of the algorithm. Since the number of agents and repetitions is constant, our Adaptive Gravity Search Algorithm (AGSA) uses ambiguous logic-based adaptation using $\varepsilon(\mathrm{k})$ instead of (in $\mathrm{g}(\mathrm{k})$ and $\varepsilon$ to $\mathrm{k}(4)$ [35]. As mentioned in the previous section of the SITO obscure module, this approach is different from the one proposed, in which an ambiguous logic block modifies the number of useful agents and g (k). In addition, our Adaptive Gravity Search Algorithm (AGSA) provides a simple ambiguous logic-based adaptation to the structure of other approaches that introduce ambiguous logic in evolutionary mechanisms. Controlling second-tier servo systems with an integrated component for optimal TS PI-FCs was discussed. em, and test results included [36]. In comparison, to provide some insight into the search performance of these algorithms, Fig. 3 and Fig. 4 illustrate the integration diagrams for all standard functions. As explained in the statistics, it is clear that for the immoral processes (F1 to F7) at the initial stage of the optimization process, the three algorithms combine in almost the same ratio [37]. Integration in the later stages of optimization. A similar conclusion can be drawn in multimodal operations IGSA to investigate at an early stage and to promote exploitation at the final stage of optimization. For low-dimensional functions, the three comparable algorithms show an equal integration rate in most of these functions because the operations are relatively simple. Exceptions are that AGSA integrates faster in F3 and F6, and AGSA offers the fastest integration rate in F9. However, all three algorithms have similar research capabilities, while GSA has better exploitation capabilities compared [38]. In comparison, to provide some insight into the search performance of these algorithms, illustrate integration maps for all key functions. As explained in the statistics, it is clear that for the immoral processes (F1 to F7) at the initial stage of the optimization process, 
the three algorithms combine in almost the same ratio [39]. However, IGSA is relatively better than GSA and AGSA in accelerating integration in the later stages of optimization. A similar conclusion can be drawn in multimodal operations IGSA to investigate at an early stage and to promote exploitation at the final stage of optimization [40]. For low-dimensional functions, the three comparable algorithms show an equal integration rate in most of these functions because the operations are relatively simple. Exceptions are that AGSA integrates faster in F3 and F6, and AGSA offers the fastest integration rate in F9. However, all three algorithms have similar research capabilities, while GSA has better exploitation capabilities compared [41].

\section{Binary gravitational search algorithm}

In this section, we look at the BGSA for binary value enhancement We describe the algorithm. The original version of the GSA was Newton's gravity and the real value inspired by the operating rules is the search engine for upgrade. Introduced the Binary Gravity Search Algorithm (BGSA) to solve problems of binary value [42]. Feature selection shows that the results of the CBIR system have been improved. Furthermore, it can be seen from the precise diagrams in that FS using BGSA is more effective than GA and BPSO. The total number of features is 270 but the number of features is 149,134 and 118 after FS using GA, BPSO and BGSA respectively [43]. So by selecting the feature, not only the accuracy is increased but also the number of features is significantly reduced. This means reducing complexity and improving performance . Such as computer vision and design recognition Feature selection is one of the most important activities in various fields [44]. In this paper, an improved version of the Binary Gravity Search Algorithm (BGSA) Proposed and intended to improve classification accuracy Used as a tool to select the best subset of features. By improving the transfer process, We provide BGSA with the ability to deal with stagnation [45]. This allows the search algorithm to explore a greater number of possibilities and avoid stagnation. Experienced in classifying some well-known databases and improving the accuracy of CBIR systems to evaluate the proposed enhanced BGSA (IBGSA). The results are compared with mechanisms such as electromagnetism [46]. The In the comparison results feature of the proposed IBGSA Confirm performance test [50]. BGSA uses interpersonal communication between agents to find the optimal solution. The forces acting on each other, the agents advance to a specific area, in which there may be better solutions, but in later optimization the different populations become dissatisfied, which can lead to entanglement in local optimism [47]. To address the undesirable effects of these deficiencies, a single agent other than the best one is chosen to enable mutation in approximately every iteration. The chosen one improves his position according to the following equation instead of the equation. Specialized agencies introduce new information to the public each time, thus increasing population diversity [48]. Although the mutation introduced above may partially alleviate the disadvantages of the algorithm, such as premature integration and entanglement in local optimism, the BGSA is a kind of intelligent optimization algorithm with its random search nature, which can have incomplete consequences [49]. To overcome the shortcoming, the BGSA should bring in some more interventions as an effective tool to lead people to better evolution and finally find the best solution. In this study, an alternative mutation strategy based on an in-depth analysis of the properties and structural controls of the units is proposed to function as this tool [50].

\section{Disruption based gravitational search algorithm}

The range of PID controller parameters was taken to be between 0 and 5. The best performance of a DGSA tuned PID controlled system is shown by comparing the system's dynamic responses and Eigen values with a GSA tuned PID controller with a load disturbance of $0.01 \mathrm{pu}$ MW. The strength of a DGSA tuned system is demonstrated GSA 9 was found to be 3.2431 in repetitions and DGSA 4 was found to be 3.2396 in repetitions [51]. It is DGSA fast and explores search space and completes the minimum value compared to GSA. We will compare ADGSA with SGSA, PSO and IGSA considering the following performance indices: standard deviation from best, worst, average and best solutions above 30 runs so far [52]. In this section, time-varying speed control is provided to better simulate the kinetic process of masses. Subsequently, an adaptive disturbance operator and levels strategy was proposed to improve exploration and exploitation capabilities and to help the masses escape from local optimism [53]. The proposed algorithm is called DGSA. In this section, we compare DGSA with other selected algorithms for average best (average-to-far), standard deviation (hierarchical-to-far), best-value (best-to-far) and worst value (worst). -So far) 30 Results obtained by running independently. Meanwhile, the ranking of algorithmic performance (i.e. average-so far) provided an overall comparison between all algorithms [54]. DGSA has a strong ability to detect global optimization in F8, F9 and F11 operations, and good capability to detect optimal proximity in F10 and F12 operations. Therefore, DGSA makes significantly better results and stronger commitment than SGSA, PSO and IGSA in research and exploitation [55]. GSA is the law of gravity And by mass interactions. Inspired horticulture is a multiagent optimization approach. The GSA approach will be used by neighboring agents Based on the movement of agents under the forces of gravity. The efficiency of agents is proportional to its exercise activity [56]. In this work, there is a drive towards the motives Is to use and establish a relationship. To create a new hassle-based GSA (DGSA), A troubleshooting operator described has been introduced. By analysis, the main problem of DGSA Between each agent and its many purposes Is to establish a relationship. To solve this problem of DGSA, an NS concept [57]. Used to separate agents into multiple layers at performance intervals. Layers are sorted And the quality of the layer to which the agent belongs determines its mass. The defined length of the external archive is also used to store non-dominant solutions [58]. In GSA, the force of gravity Leads two solutions It is useless to move too close to each other, More research in the search space It is better to move one of them in order to upgrade. In case of pre-integration, There is no analytical capacity one astronomical based 
disturbance operator has been added to improve the flexibility of GSA [59]. Interference-based Gravity To solve STHTS problems Search Algorithm (DGSA) proposed. Proposed DGSA approach Its study and Helps to increase the flexibility of exploitation skills. To simulate the proposed DGSA, the ideal solution (massive object, $\mathrm{M}$ ) is considered to be the star of the system, and other solutions may disintegrate and scatter in space under the star's gravitational pull (Sarafrasi, Nejmabadipour, \& Saryasti). If all solutions except the best solution satisfy the disturbance level, these solutions will be disrupted [60].

\section{Conclusion}

Some optimization problems are two possible values, zero and there are variables with one. These issues are handled by binary versions. Introduced the Binary Gravity Search Algorithm (PGSA). In the binary context, each dimension has a value of 0 or 1 . Moving in each dimension means that the corresponding value is changed from 0 to 1 or vice versa. In BGSA, the equations for force and speed upgrade are the same as in the continuous version, and the Hamming distance is used to calculate the $\mathrm{XRP}$ distance. A control The optimization problem is mathematically defined as Eq. Under the Ng number of equation control functions Development of an objective process And this problem is caused by the Nh number of equation control functions expressed by $\mathrm{g}$ and $\mathrm{h}$ in the equation. Respectively. To deal with restrictions, It is common practice to impose punishment for objective action. To evaluate the solution $\mathrm{X}$ in the reduction problem Penalty functions are included in the objective process. GSA was inspired by Newton's theory: "Every particle in the universe attracts other particles with a force that is directly proportional to the product of their masses and inversely proportional to the square of the distance between them". Gravity is a force that pulls all objects together. The original version of GSA was designed to search for truly valuable issues are set in the binary separate encryption is introduced. In an earlier version of the GSA, the algorithm "gravity keys" Multi-dimensional continuous space (search location) Causing direct changes in the position of search points. Effect of these keys is on the binary vector Converts the probability value to each element, It guides whether those elements get a value of 0 or 1 . GSA is a new multi-agent optimization. Algorithm derived from the law of general attraction. Algorithm gravity keys Based on the motion of certain particles under influence, Others use. Hasanzade and Romani are GSA's Proposed the first multipurpose variant, This is Canyon proposed to update the external archive to neighboring countries. is updated at intervals. Such as computer vision and design recognition features aimed at improving classification accuracy Used as a tool to select the best subgroup. By improving the transfer process, We provide BGSA with the ability to deal with stagnation. This allows the search algorithm to explore a greater number of possibilities and avoid stagnation. And in classifying some well-known datasets Experienced to evaluate the proposed enhanced BGSA (IBGSA). The results are compared with mechanisms such as the Electromagnetism. IBGSA proposed in the feature test Performance comparison results confirm. GSA is horticulture inspired by the law of gravity and mass interactions [18] Is the multi-agent optimization approach. The GSA approach will be used by neighboring agents Based on the movement of agents under the forces of gravity. The efficiency of agents is measured by their masses, And the weight of each agent is proportional to its exercise activity. There is a drive towards the use and establish a relationship between its conflicting purposes. To create a new hassle-based GSA (DGSA), [28] A troubleshooting operator described in has been introduced. By analysis, the main problem of DGSA is for each agent Is to establish a relationship between its many purposes. To solve in the performance interval Used to separate agents into multiple layers. The defined length of the external archive is also used to store nondominant solutions.

\section{Reference}

[1]. Rashedi, Esmat, Elaheh Rashedi, and Hossein Nezamabadi-pour. "A comprehensive survey on gravitational search algorithm." Swarm and evolutionary computation 41 (2018): 141-158.

[2]. Sarafrazi, Soroor, Hossein Nezamabadi-pour, and Saeid Saryazdi. "Disruption: a new operator in gravitational search algorithm." Scientia Iranica 18, no. 3 (2011): 539-548.

[3]. Wang, Yirui, Yang Yu, Shangce Gao, Haiyu Pan, and Gang Yang. "A hierarchical gravitational search algorithm with an effective gravitational constant." Swarm and Evolutionary Computation 46 (2019): 118-139.

[4]. Li, Chaoshun, and Jianzhong Zhou. "Parameters identification of hydraulic turbine governing system using improved gravitational search algorithm." Energy Conversion and Management 52, no. 1 (2011): 374-381.

[5]. Jiang, Shanhe, Yan Wang, and Zhicheng Ji. "Convergence analysis and performance of an improved gravitational search algorithm." Applied Soft Computing 24 (2014): 363-384.

[6]. Rashedi, Esmat, Hossein Nezamabadi-Pour, and Saeid Saryazdi. "BGSA: binary gravitational search algorithm." Natural Computing 9, no. 3 (2010): 727-745.

[7]. Sahoo, G. "A review on gravitational search algorithm and its applications to data clustering \& classification." IJ Intelligent Systems and Applications 6 (2014): 79-93.

[8]. Sahu, Rabindra Kumar, Sidhartha Panda, and Saroj Padhan. "A novel hybrid gravitational search and pattern search algorithm for load frequency control of nonlinear power system." Applied Soft Computing 29 (2015): 310-327.

[9]. Hassanzadeh, Hamid Reza, and Modjtaba Rouhani. "A multi-objective gravitational search algorithm." In 2010 2nd international conference on computational intelligence, communication systems and networks, pp. 7-12. IEEE, 2010 . 
[10]. Hemmatian, Hossein, Abdolhossein Fereidoon, and Ehsanolah Assareh. "Optimization of hybrid laminated composites using the multi-objective gravitational search algorithm (MOGSA)." Engineering Optimization 46, no. 9 (2014): 1169-1182.

[11]. Ramachandran, M., Sahas Bansal, Vishal Fegade, and Pramod Raichurkar. "Analysis of bamboo fibre composite with polyester and epoxy resin." International Journal on Textile Engineering \& Processes 1, no. 4 (2015): 18-21.

[12]. Fegade, V. T., and Kiran S. Bhole. "Finite Element Analysis and Material Optimization for Equivalent Strength of Composite Connecting Rod." SSRG International Journal of Mechanical Engineering (SSRG-IJME) 2, no. 2 (2015).

[13]. Abbasian, Mohammad Amir, Hossein Nezamabadi-pour, and Maryam Amoozegar. "A clustering based archive multi objective gravitational search algorithm." Fundamenta Informaticae 138, no. 4 (2015): 387-409.

[14]. Nobahari, Hadi, Mahdi Nikusokhan, and Patrick Siarry. "A multi-objective gravitational search algorithm based on non-dominated sorting." International Journal of Swarm Intelligence Research (IJSIR) 3, no. 3 (2012): 32-49.

[15]. Kumar, J. Vijaya, DM Vinod Kumar, and K. Edukondalu. "Strategic bidding using fuzzy adaptive gravitational search algorithm in a pool based electricity market." Applied soft computing 13, no. 5 (2013): 2445-2455.

[16]. Precup, Radu-Emil, Radu-Codrut David, Emil M. Petriu, Stefan Preitl, and Mircea-Bogdan Rădac. "Fuzzy logicbased adaptive gravitational search algorithm for optimal tuning of fuzzy-controlled servo systems." IET Control Theory \& Applications 7, no. 1 (2013): 99-107.

[17]. Rashedi, Esmat, and Hossein Nezamabadi-pour. "Improving the precision of CBIR systems by feature selection using binary gravitational search algorithm." In The 16th CSI International Symposium on Artificial Intelligence and Signal Processing (AISP 2012), pp. 039-042. IEEE, 2012.

[18]. Yuan, Xiaohui, Bin Ji, Shuangquan Zhang, Hao Tian, and Yanhong Hou. "A new approach for unit commitment problem via binary gravitational search algorithm." Applied Soft Computing 22 (2014): 249-260.

[19]. Sharma, Veena, R. Naresh, and Harish Pulluri. "Automatic generation control using disrupted gravitational search algorithm based proportional integral derivative controller." In 2015 2nd International Conference on Recent Advances in Engineering \& Computational Sciences (RAECS), pp. 1-6. IEEE, 2015.

[20]. Ding, GuiYan, DaQin Zhang, and Hao Liu. "An adaptive disruption based gravitational search algorithm with time-varying velocity limitation." In 2016 35th Chinese Control Conference (CCC), pp. 9201-9206. IEEE, 2016.

[21]. Nadakuditi, Gouthamkumar, Veena Sharma, and Ram Naresh. "Non-dominated sorting disruption-based gravitational search algorithm with mutation scheme for multi-objective short-term hydrothermal scheduling." Electric Power Components and Systems 44, no. 9 (2016): 990-1004.

[22]. Gouthamkumar, N., Veena Sharma, and Ram Naresh. "Disruption based gravitational search algorithm for short term hydrothermal scheduling." Expert Systems with Applications 42, no. 20 (2015): 7000-7011.

[23]. Amol Lokhande, C. Venkateswaran, M. Ramachandran, C. Vidhya, R. Kurinjimalar. " A Study on Various Implications on Reusing in Manufacturing", REST Journal on Emerging trends in Modelling and Manufacturing, 7(2), (2021): 63-69.

[24]. Shanmugasundar, G., R. Sivaramakrishnan, R. Sridhar, and M. Rajmohan. "Computer aided modelling and static analysis of an inspection robot." In Applied Mechanics and Materials, vol. 766, pp. 1055-1060. Trans Tech Publications Ltd, 2015.

[25]. C. Venkateswaran; M. Ramachandran; M. Amudha; T. Vennila; M. Manjula "A Review on Differential Evolution Optimization Techniques", Data Analytics and Artificial Intelligence, 1(1), (2021):24-31.

[26]. Shanmugasundar, G., P. Jagadeeshwar, S. Adithya, V. Nagappan, and M. Bhaskar. "Design, fabrication and analysis of personal vacuum assisted climber." In Journal of Physics: Conference Series, vol. 1362, no. 1, p. 012057. IOP Publishing, 2019.

[27]. Amol Lokhande, C. Venkateswaran, M. Ramachandran, S. Chinnasami, T. Vennila."A Review on Various Implications on Re engineering in Manufacturing", REST Journal on Emerging trends in Modelling and Manufacturing, 7(3), 2021:70-75.

[28]. Amol Lokhande; C. Venkateswaran, M. Ramachandran, C. Sathiyaraj, K. Nathiya, "Recycling Process Impact in Current Scenario Manufacturing A Study", Recent trends in Management and Commerce, 2(1), (2021):20-25.

[29]. C. Venkateswaran; M. Ramachandran; Vimala saravanan; T. Vennila " A Study on Artificial intelligence with Machine learning and Deep Learning Techniques", Data Analytics and Artificial Intelligence, 1(1), (2021):32-37.

[30]. Shanmugasundar, G., M. Vanitha, Robert Čep, Vikas Kumar, Kanak Kalita, and M. Ramachandran. "A Comparative Study of Linear, Random Forest and AdaBoost Regressions for Modeling Non-Traditional Machining." Processes 9, no. 11 (2021): 2015.

[31]. Nisha Sherief; M. Anand; M. Ramachandran; Vidhya Prasanth, "A Review on Various Biofuels and its Applications", REST Journal on Emerging trends in Modelling and Manufacturing, 8(1), (2022):1-9. 
[32]. Fegade, Vishal, Kshitij Srivastava, A. V. Kale, and Rajiv K. Srivastava. "Feasibility analysis of design for remanufacturing in bearing using hybrid fuzzy-topsis and taguchi optimization." Independent Journal of Management \& Production 11, no. 1 (2020): 81-95.

[33]. Vimala Saravanan; M. Ramachandran; T. Vennila; G. Mathivanan "A Study on Multi-Objective Optimization on the basis of Ratio Analysis", Recent trends in Management and Commerce, 2(3), (2021):16-22

[34]. S. Chinnasami, M. Ramachandran, P. Vidhya, M. Gowri " Study of Evaluation Based on Distance from Average Solution on Moyamoya Disease and Energy application" REST Journal on Emerging trends in Modelling and Manufacturing, 7(4), (2022):116-124.

[35]. Krishnan, G. Sai, G. Shanmugasundar, M. Vanitha, and N. Sivashanmugam. "Mechanical properties of chemically treated Banana and ramie fibre reinforced polypropylene composites." In IOP Conference Series: Materials Science and Engineering, vol. 961, no. 1, p. 012013. IOP Publishing, 2020.

[36]. R. Kurinjimalar, S. Vimala, M. Silambarasan, S. Chinnasami. "A Review on Coir fibre Reinforced Composites with Different Matrix", REST Journal on Emerging trends in Modelling and Manufacturing, 7(2), (2021):25-32.

[37]. Krishnan, G. Sai, G. Shanmugasundar, M. Vanitha, S. Srinivasan, and G. Suresh. "Investigation on the Mechanical and Morphological Properties of Red banana/Ramie Fiber vinyl ester composites." In IOP Conference Series: Materials Science and Engineering, vol. 961, no. 1, p. 012015. IOP Publishing, 2020.

[38]. Lokhande, Dr Amol, Dr C. Venkateswaran, Dr M. Ramachandran, C. Vidhya, and R. Kurinjimalar. "A Study on Various Implications on Reusing in Manufacturing." REST Journal on Emerging trends in Modelling and Manufacturing 7, no. 2 (2021).

[39]. Shanmugasundar, G., and R. Sivaramakrishnan. "Software Development for an Inverse Kinematics of SevenDegrees of Freedom Newly Designed Articulated Inspection Robot." International Journal of Computer Applications 975 (2012): 8887.

[40]. Rashedi, Esmat, and Hossein Nezamabadi-pour. "Feature subset selection using improved binary gravitational search algorithm." Journal of Intelligent \& Fuzzy Systems 26, no. 3 (2014): 1211-1221.

[41]. Shanmugasundar, G., R. Sivaramakrishnan, and S. Venugopal. "Modeling, Design and Static Analysis of Seven Degree of Freedom Articulated Inspection Robot." In Advanced Materials Research, vol. 655, pp. 1053-1056. Trans Tech Publications Ltd, 2013.

[42]. Kalita, Kanak, Uvaraja Ragavendran, Manickam Ramachandran, and Akash Kumar Bhoi. "Weighted sum multiobjective optimization of skew composite laminates." Structural Engineering and Mechanics 69, no. 1 (2019): 21 31.

[43]. Shanmugasundar, G., R. Sivaramakrishnan, and S. Balasubramani. "Method of Trajectory Generation of a Generic Robot using Bresenham's Circle Algorithm." Indian Journal of Science and Technology 9, no. 48 (2016): 1-6.

[44]. Kittur, Jeevan, M. RAMACHANDRAN, Vishal Fegade, and U. Ragavendran. "Numerical Investigation of Total Deformation Inroller Bearing using Ansys Analysis." International Journal of Mechanical and Production Engineering Research and Development (IJMPERD): 51-58.

[45]. Ramachandran, M., U. Ragavendran, and Vishal Fegade. "Selection of Used Piston for Remanufacturing Using Fuzzy TOPSIS Optimization." In Fuzzy Systems and Data Mining IV, pp. 61-67. IOS Press, 2018.

[46]. Fegade, Vishal, Shannay Rawal, and M. Ramachandran. "Metamodel-based parametric study of composite laminates." In IOP Conference Series: Materials Science and Engineering, vol. 810, no. 1, p. 012051. IOP Publishing, 2020.

[47]. Soniya Sriram, M. Ramachandran, Sathiyaraj Chinnasamy, G. Mathivanan "A Review on Multi-Criteria DecisionMaking and Its Application", REST Journal on Emerging trends in Modelling and Manufacturing, 7(4), (2022):101107.

[48]. Ramachandran, M., Vishal Fegade, and U. Ragavendran. "Parameters Optimisation For Drilling Of Austenitic Stainless Steel By Taguchi Method Using Desirability Function Analysis." Technology 8, no. 11 (2017): 229-237.

[49]. Ragavendran, U., Viral Mehta, Vishal Fegade, and M. Ramachandran. "Dynamic Analysis of Single Fold Symmetric Composite Laminates." international Journal of civil Engineering and Technology 8, no. 11 (2017): 536545.

[50]. Ramachandran, M., Vishal Fegade, and P. P. Raichurkar. "Strategy Performance Evaluation of a Port Organisation based on Multi-Criteria Decision Making using Fuzzy Logic Method." NMIMS Management Review 33 (2017): 27 34.

[51]. Fegade, Vishal, Gajanan Jadhav, and M. Ramachandran. "Design, Modelling and Analysis of Tilted Human Powered Vehicle." In IOP Conference Series: Materials Science and Engineering, vol. 377, no. 1, p. 012215. IOP Publishing, 2018. 
[52]. Shanmugasundar, G., R. Sivaramakrishnan, and M. Rajmohan. "Computer aided simulation for workspace plot of a newly designed inspection robot." In 2014 IEEE International Conference on Computational Intelligence and Computing Research, pp. 1-6. IEEE, 2014.

[53]. Vikrant Sharma; M. Ramachandran; Sathiyaraj Chinnasamy; Vimala Saravanan, " A Review on Structural Equation Modeling and Its Classification" REST Journal on Emerging trends in Modelling and Manufacturing, 7(4), (2022): 135-142

[54]. Gadde Mehar Chaitanya, M.P.Jenarthanan, C. Sathiyaraj, "A Review on Glass fibre Reinforced Composites with Different Matrix", REST Journal on Emerging trends in Modelling and Manufacturing, 7(1), (2021):18-24.

[55]. Manickam, Ramachandran. "Back propagation neural network for prediction of some shell moulding parameters." Periodica Polytechnica Mechanical Engineering 60, no. 4 (2016): 203-208.

[56]. Ramachandran, M., Sahas Bansal, and Pramod Raichurkar. "Scrutiny of jute fiber poly-lactic acid (PLA) resin reinforced polymeric composite." Journal of the Textile Association 76, no. 6 (2016): 372-375.

[57]. Pradeep, P., J. Edwin Raja Dhas, M. Ramachandran, and B. Stanly Jones Retnam. "Mechanical Characterization of jute fiber over glass and carbon fiber reinforced polymer composites." International Journal of Applied Engineering Research 10, no. 11 (2015): 10392-10396.

[58]. Sompura, P., and V. Karnad. "POLYESTER-Product development from spun silk/polyester fabrics printed with geometric motifs and newsprints for home interiors." Colourage 58, no. 3 (2011): 40.

[59]. Mulchandani, Neha, and Vishaka Karnad. "Application of zinc oxide nano particles using polymeric binders on cotton fabric." Research Journal of Textile and Apparel (2021).

[60]. Shanmugasundar, G., R. Sivaramakrishnan, R. Sridhar, and M. Rajmohan. "Computer aided modelling and static analysis of an inspection robot." In Applied Mechanics and Materials, vol. 766, pp. 1055-1060. Trans Tech Publications Ltd, 2015. 